\title{
A Semi-Induced Subgraph Characterization of Upper Domination Perfect Graphs
}

\author{
Igor E. Zverovich \\ Faculty of Mechanics and Mathematics \\ Belarus State University, Minsk 220050 \\ Belarus
}

\author{
Vadim E. Zverovich* \\ Department II of Mathematics \\ RWTH Aachen, Aachen 52056 \\ Germany
}

\begin{abstract}
Let $\beta(G)$ and $\Gamma(G)$ be the independence number and the upper domination number of a graph $G$, respectively. A graph $G$ is called $\Gamma$-perfect if $\beta(H)=\Gamma(H)$, for every induced subgraph $H$ of $G$. The class of $\Gamma$-perfect graphs generalizes such well-known classes of graphs as strongly perfect graphs, absorbantly perfect graphs, and circular arc graphs. In this article, we present a characterization of $\Gamma$-perfect graphs in terms of forbidden semi-induced subgraphs. Key roles in the characterization are played by the odd prism and the even Möbius ladder, where the prism and the Möbius ladder are well-known 3-regular graphs [2]. Using the semi-induced subgraph characterization, we obtain a characterization of $K_{1,3}$-free $\Gamma$-perfect graphs in terms of forbidden induced subgraphs. J. Graph Theory 31 (1999), 29-49
\end{abstract}

Keywords: upper domination perfect graphs; semi-induced subgraph characterization

\section{Introduction}

All graphs will be finite and undirected without multiple edges. Unless otherwise stated, all graphs have no loops. If $G$ is a graph, $V(G)$ denotes the set, and $|G|$ the number, of vertices in $G$. Let $N(x)$ denote the neighborhood of a vertex $x$, and let $\langle X\rangle$ denote the subgraph of $G$ induced by $X \subseteq V(G)$. Also let $N(X)=\cup_{x \in X} N(x)$ and $N[X]=N(X) \cup X$. Denote by $\delta(G)$ the minimal degree of vertices in $G$. A path, a cycle and a complete graph of order $n$ will be denoted by $P_{n}, C_{n}$ and $K_{n}$, respectively.

A set $I \subseteq V(G)$ is called independent if no two vertices of $I$ are adjacent. A set $X$ is called a dominating set if $N[X]=V(G)$. An independent dominating set is a vertex subset that is both independent and dominating, or equivalently, is maximal independent. The independence number $\beta(G)$ is the maximum cardinality of a (maximal) independent set of $G$, and the independent domination number $i(G)$ is the minimum cardinality taken over all maximal independent sets of $G$. The domination number $\gamma(G)$ is the minimum

\footnotetext{
*On leave from Faculty of Mechanics and Mathematics, Belarus State University, Minsk 220050, Belarus. Supported by the Alexander von Humboldt Foundation.
} 
cardinality of a (minimal) dominating set of $G$, and the upper domination number $\Gamma(G)$ is the maximum cardinality taken over all minimal dominating sets of $G$. For $x \in X$, the set

$$
P N(x, X)=N[x]-N[X-\{x\}]
$$

is called the private neighborhood of $x$. If $P N(x, X)=\emptyset$, then $x$ is said to be redundant in $X$. A set $X$ containing no redundant vertex is called irredundant. The irredundance number $\operatorname{ir}(G)$ is the minimum cardinality taken over all maximal irredundant sets of $G$, and the upper irredundance number $\operatorname{IR}(G)$ is the maximum cardinality of a (maximal) irredundant set of $G$.

The following relationship among the parameters under consideration is well-known $[7,9]$ :

$$
\operatorname{ir}(G) \leq \gamma(G) \leq i(G) \leq \beta(G) \leq \Gamma(G) \leq I R(G)
$$

Definition 1 A graph $G$ is called irredundance perfect (ir-perfect) if $\operatorname{ir}(H)=\gamma(H)$, for every induced subgraph $H$ of $G$.

Definition 2 A graph $G$ is called domination perfect ( $\gamma$-perfect) if $\gamma(H)=i(H)$, for every induced subgraph $H$ of $G$.

Definition 3 A graph $G$ is called upper domination perfect ( $\Gamma$-perfect) if $\beta(H)=\Gamma(H)$, for every induced subgraph $H$ of $G$.

Definition 4 A graph $G$ is called upper irredundance perfect (IR-perfect) if $\Gamma(H)=$ $I R(H)$, for every induced subgraph $H$ of $G$.

The classes of upper domination perfect graphs and upper irredundance perfect graphs in a sense are dual to the classes of domination perfect graphs and irredundance perfect graphs, respectively. A lot of interesting results on domination perfect graphs $[1,3,12,16$, $17,24,27,28,29,31,33]$ and irredundance perfect graphs $[3,4,10,20,21,25,26,32]$ are known. A finite induced subgraph characterization of the entire class of domination perfect graphs was recently obtained in [33], while the problems of characterizing the entire class of irredundance perfect graphs and upper irredundance perfect graphs are still open. For a short survey on domination perfect graphs, see also [33].

We summarize the known results on $\Gamma$-perfect and $I R$-perfect graphs. The following important theorem gives the relationship between the class of $\Gamma$-perfect graphs and the class of $I R$-perfect graphs.

Theorem A (Gutin and Zverovich [14]) Any $\Gamma$-perfect graph is IR-perfect.

Thus, $\Gamma$-perfect graphs form a subclass of $I R$-perfect graphs. On the other hand, a number of well-known classes of graphs are subclasses of $\Gamma$-perfect graphs, and consequently, $I R$-perfect graphs. Cockayne et al. [7] proved that bipartite graphs are $\Gamma$-perfect, and Jacobson and Peters [23] showed that chordal graphs are $\Gamma$-perfect. The next theorem generalizes these results, since bipartite graphs and chordal graphs are strongly perfect graphs. Recall that a graph $G$ is called strongly perfect if every induced subgraph $H$ of $G$ has a stable transversal, where a stable transversal $S$ of $H$ is a vertex subset of $H$ such that $|S \cap C|=1$ for any maximal clique $C$ of $H$. 
Theorem B (Cheston and Fricke [5], Jacobson and Peters [22]) A strongly perfect graph is $\Gamma$-perfect.

It may be pointed out that, besides bipartite and chordal graphs, strongly perfect graphs contain comparability graphs, perfectly orderable graphs, peripheral graphs, complements of chordal graphs, Meyniel graphs, parity graphs, $i$-triangulated graphs, permutation graphs, cographs, and hence all these classes are subclasses of $\Gamma$-perfect graphs. Hammer and Maffray [15] defined a graph $G$ to be absorbantly perfect if every induced subgraph $H$ of $G$ contains a minimal dominating set that meets all maximal cliques of $H$. It turned out that absorbantly perfect graphs are $\Gamma$-perfect (Theorem $\mathrm{C}$ ). Since every strongly perfect graph is absorbantly perfect, we see that Theorem B follows from the more general Theorem C.

Theorem C (Gutin and Zverovich [14]) An absorbantly perfect graph is $\Gamma$-perfect.

A graph is called circular arc if it can be represented as the intersection graph of arcs on a circle.

Theorem D (Golumbic and Laskar [13]) A circular arc graph is $\Gamma$-perfect.

The following theorem gives a sufficient condition for a graph to be $I R$-perfect.

Theorem E (Cockayne, Favaron, Payan and Thomason [7]) If a graph $G$ does not contain $P_{5}, C_{5}, \operatorname{Pr}_{3}-v_{1}$ and $\operatorname{Pr}_{3}-v_{1}-v_{2} v_{3}$ as induced subgraphs, where $\operatorname{Pr}_{3}$ is shown in Fig. 1, then $G$ is IR-perfect.

Using Theorem A we see that Theorem F improves Theorem E.

Theorem F (Gutin and Zverovich [14]) If a graph $G$ does not contain $P_{5}$ and $\mathrm{Pr}_{3}$ in Fig. 1 as induced subgraphs, then $G$ is $\Gamma$-perfect.

Other sufficient conditions for a graph to be $\Gamma$-perfect or $I R$-perfect can be found in $[10,14,23,30]$, one of them is stated in Corollary 2. A number of authors $[6,7,8,11]$ investigated graphs $G$ having $\beta(G)=\Gamma(G)$, i.e., these parameters are not necessarily equal for a proper induced subgraph of $G$. Cockayne et al. [8] proved that

$$
\beta(G)=\Gamma(G)=I R(G)
$$

for the representative graph of any hereditary hypergraph. Cheston et al. [6] showed that this equality is valid for upper bound graphs which extend the class of representative graphs of hereditary hypergraphs, while Fellows et al. [11] proved that the same equality holds for trestled graphs. It was also shown by Cheston et al. [6] that $\beta(G)=\Gamma(G)$ for simplicial graphs, which generalize the class of upper bound graphs.

In this article, we introduce the concept of a semi-induced subgraph (Definition 8), and we present two characterizations of the entire class of $\Gamma$-perfect graphs in terms of forbidden semi-induced subgraphs (Theorems 1 and 2). Key roles in the characterizations are played by the odd prism and the even Möbius ladder, where the prism and the Möbius ladder are well-known 3-regular graphs [2]. Using the semi-induced subgraph characterization of $\Gamma$ perfect graphs, we obtain a result of Jacobson and Peters [22] on $\Gamma$-perfect graphs (Corollary 1) and also a characterization of $K_{1,3}$-free $\Gamma$-perfect graphs in terms of forbidden induced 
subgraphs. The latter result implies a known sufficient condition for a $K_{1,3}$-free graph to be $\Gamma$-perfect (Corollary 2). Notice here that $K_{1,3}$-free graphs are $\gamma$-perfect [1], and that $K_{1,3}$-free $i r$-perfect graphs were characterized by Favaron [10], who also found a sufficient condition for a $K_{1,3}$-free graph to be $I R$-perfect.

\section{Basic Definitions}

We need the following definitions.

Definition 5 Two vertex subsets $A, B$ of a graph $G$ independently match each other if $A \cap B=\emptyset,|A|=|B|$, and all edges between $A$ and $B$ form a perfect matching in $\langle A \cup B\rangle$.

Definition 6 A graph $G$ of order $2 k$ is called a W-graph if there is a partition $V(G)=$ $A \cup B$ such that $A$ and $B$ independently match each other. Clearly, $|A|=|B|=k$. The sets $A$ and $B$ are called parts, and the graph $G$ is denoted by $G(A, B)$.

It is not difficult to see that a $\mathrm{W}$-graph may have several partitions into parts. Hence a W-graph is considered in Sections 2 and 3 together with a fixed partition into parts.

Definition 7 Let $G$ be a $W$-graph, $G=G(A, B)$. Edges between the parts $A$ and $B$ are called b-edges and denoted in our figures by bold lines. Edges which are not b-edges are called l-edges and denoted by thin lines.

We can understand the above partition of the edge set as a coloring of the edge set with two colors 'b' and 'l'. Note that if the set $E_{b}$ of b-edges of a connected W-graph $G$ is given, then there is only one partition $V(G)=A \cup B$ such that $A$ and $B$ independently match each other, i.e., $G=G(A, B)$ and $E_{b}$ is the set of b-edges with respect to this partition.

Definition 8 Let $H=H(A, B)$ be a $W$-graph with parts $A$ and $B$. The graph $H$ is called a semi-induced subgraph of a graph $G$ if $H$ is a subgraph of $G$, and in the graph $G$ the sets $A$ and $B$ independently match each other.

In other words, let $A$ and $B$ independently match each other in $G$ and let $P$ be the perfect matching between $A$ and $B$ in $\langle A \cup B\rangle$. If $E_{1} \subseteq E\langle A\rangle$ and $E_{2} \subseteq E\langle B\rangle$, then the graph $H$ having $V(H)=A \cup B$ and $E(H)=E_{1} \cup E_{2} \cup P$ is a semi-induced subgraph of $G$. Thus, any semi-induced subgraph of a graph is a W-graph, and if $H$ is not a W-graph, then $G$ cannot contain $H$ as a semi-induced subgraph.

Definition 9 A graph $G$ is called a bl-graph if a partition of the set $E(G)$ into the set of b-edges (bold) and l-edges (thin) is given, provided that the set of b-edges forms a matching in $G$. If the b-edges form a perfect matching, then $G$ is called a perfect bl-graph. For example, any $W$-graph is a perfect bl-graph. An even (odd) bl-graph has the even (odd) number of b-edges.

Definition 10 A simple bl-chain $P$ is called alternating if for any two consecutive edges of $P$ one of them is a b-edge and another is an l-edge. The alternating simple chain $P$ is called $a$ b-chain (l-chain) if the end edges of $P$ are b-edges (l-edges). Clearly, b-chains and l-chains always have even order. If we identify the end vertices $u_{1}$ and $u_{2 n}$ in the l-chain $\left(u_{1}, u_{2}, \ldots, u_{2 n}\right)$, where $n \geq 2$, then we obtain the simple cycle $\left(u_{1}, u_{2}, \ldots, u_{2 n-1}\right)$ which is called an l-cycle starting with $u_{1}$. 
Definition 11 For a perfect bl-graph $G$ we define the operation of W-reducibility as follows. Each vertex $u \in V(G)$ is labeled by $c(u) \in\{A, B\}$. Further, each edge $e=v w \in E(G)$ is replaced by an alternating bl-chain $P_{e}$ with end vertices $v, w$ in accordance with the next rule:

- If $e$ is an l-edge and $c(v)=c(w)$, then $P_{e}$ is an even l-chain.

- If $e$ is an l-edge and $c(v) \neq c(w)$, then $P_{e}$ is an odd l-chain.

- If $e$ is a b-edge and $c(v)=c(w)$, then $P_{e}$ is an even b-chain.

- If $e$ is a b-edge and $c(v) \neq c(w)$, then $P_{e}$ is an odd b-chain.

Definition 12 The prism $\operatorname{Pr}_{n}(n \geq 3)$ consists of two disjoint cycles

$$
C_{1}=\left(u_{1}, u_{2}, \ldots, u_{n}\right), \quad C_{2}=\left(v_{1}, v_{2}, \ldots, v_{n}\right)
$$

and the remaining edges are of the form $u_{i} v_{i}, 1 \leq i \leq n$. The prism $\operatorname{Pr}_{1}$ is two loops connected by the edge $u_{1} v_{1}$, this is the only case where loops are permitted. If the prism $\mathrm{Pr}_{n}$ is considered as a perfect bl-graph, then its set of b-edges is $\left\{u_{i} v_{i}: 1 \leq i \leq n\right\}$.

Definition 13 The Möbius ladder $M l_{n}$ is constructed from the cycle $C=\left(u_{1}, u_{2}, \ldots, u_{2 n}\right)$ by adding the edges $u_{i} u_{n+i}(1 \leq i \leq n)$ joining each pair of opposite vertices of $C$. If the Möbius ladder $M l_{n}$ is considered as a perfect bl-graph, then its set of b-edges is $\left\{u_{i} u_{n+i}\right.$ : $1 \leq i \leq n\}$

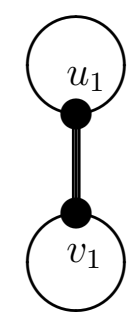

$\operatorname{Pr}_{1}$

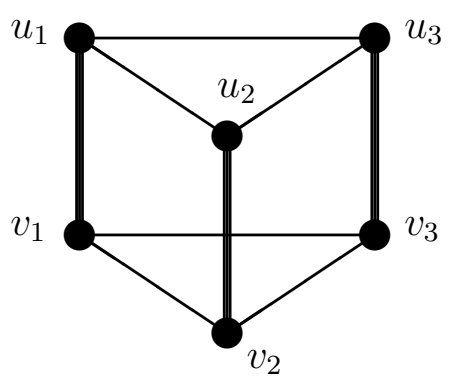

$\mathrm{Pr}_{3}$

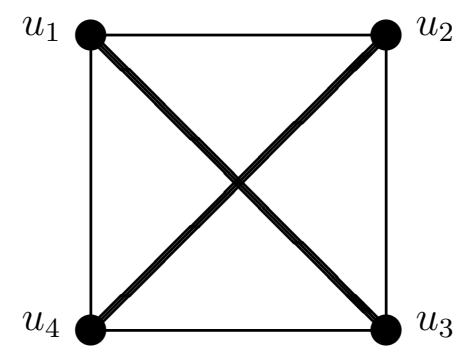

$\mathrm{Ml}_{2}$

FIGURE 1. Odd prisms $\operatorname{Pr}_{1}, \operatorname{Pr}_{3}$ and even Möbius ladder $M l_{2}$.

The odd prisms $\mathrm{Pr}_{1}$ and $\mathrm{Pr}_{3}$ and the even Möbius ladder $M l_{2}$ are shown in Fig. 1. The odd prisms and the even Möbius ladders play a key role in the definition of basis graphs.

Definition 14 A graph $G$ without loops is called a basis if it can be obtained from the odd prism $\operatorname{Pr}_{2 n+1}(n \geq 0)$ or the even Möbius ladder $M_{2 m}(m \geq 1)$ by the operation of W-reducibility.

We will prove later that a basis graph is a W-graph whose perfect matching between the parts consists of b-edges determined by the operation of W-reducibility. A basis graph $G$ cannot have loops. Hence, if $G$ is obtained from $P r_{1}$, then every loop (l-edge) of $P r_{1}$ must be replaced in accordance with Definition 11 by an alternating even l-cycle (l-chain with equal end vertices) having at least two b-edges. 


\section{Characterization of $Г$-Perfect Graphs}

The following theorem gives a characterization of upper domination perfect graphs in terms of forbidden semi-induced subgraphs.

Theorem 1 A graph $G$ is a $\Gamma$-perfect graph if and only if $G$ does not contain any basis graph as a semi-induced subgraph.

Proof: The proof of Theorem 1 is based on 11 lemmas.

Lemma 1 If $G$ is a $W$-graph of order $2 k$, then

$$
\Gamma(G)=k \geq \beta(G) .
$$

Proof: Any independent set of $G$ contains at most one vertex of each b-edges, and hence $\beta(G) \leq k$. Since $A$ is a minimal dominating set, we have $\Gamma(G) \geq k$. Let us prove that $\Gamma(G) \leq k$. Let $D$ be a minimal dominating set of $G$ of cardinality $\Gamma(G)$. If $\operatorname{deg}_{\langle D\rangle} d>0$ for $d \in D$, then there is a vertex $f \in V(G)-D$ such that $N(f) \cap D=\{d\}$. If $\operatorname{deg}_{\langle D\rangle} d=0$ for $d \in D$, then there is a vertex $f$ such that $d f$ is a b-edge. Obviously $f \in V(G)-D$. Thus, for each vertex $d \in D$ we can indicate a vertex $f$ from $V(G)-D$ and evidently that different vertices of $D$ result in different vertices of $V(G)-D$, i.e., $|D| \leq|V(G)-D|$. We have $\Gamma(G)=|D| \leq k$.

Definition $15 A$-graph $G$ of order $2 k$ is called strong if

$$
\beta(G)<k \text {. }
$$

Lemma $2 A$ graph $G$ is $\Gamma$-perfect if and only if $G$ does not contain any strong graph as an induced subgraph.

Proof: The necessity follows from the fact that for a strong graph $H, \beta(H)<\frac{1}{2}|H|$, while $\Gamma(H)=\frac{1}{2}|H|$ by Lemma 1 . To prove the sufficiency, let $G^{\prime}$ be an arbitrary induced subgraph of $G$, and let $D$ be a minimal dominating set in $G^{\prime}$ of cardinality $\Gamma\left(G^{\prime}\right)$. Denote by $J$ the set of all isolated vertices in $\langle D\rangle$, i.e. $J=\left\{v \in D: \operatorname{deg}_{\langle D\rangle} v=0\right\}$, and let $A=D-J$. Since $D$ is a minimal dominating set, it follows that for each vertex $a \in A$ there is a vertex $b \notin D$ such that $N(b) \cap D=\{a\}$. Taking such a vertex $b$ for each $a \in A$, we define $B$ as the union of these vertices. The graph $H=\langle A \cup B\rangle$ is obviously a $\mathrm{W}$-graph with parts $A$ and $B$, and $|A|=|B|=k$. Since $H$ cannot be strong, we have $\beta(H) \geq k$. Let $I$ be an independent set of $H$ of cardinality $k$. It is evident that the set $I \cup J$ is independent in $G^{\prime}$. Consequently,

$$
\beta\left(G^{\prime}\right) \geq|I|+|J|=|A|+|J|=|D|=\Gamma\left(G^{\prime}\right) .
$$

Since $\beta\left(G^{\prime}\right) \leq \Gamma\left(G^{\prime}\right)$, we have $\beta\left(G^{\prime}\right)=\Gamma\left(G^{\prime}\right)$. Thus, the graph $G$ is $\Gamma$-perfect.

Definition 16 A connected strong graph $G(A, B)$ of order $2 k$ is called critical if $\delta(G) \geq 2$ and for any l-edge $e \in E(G)$,

$$
\beta(G-e)=k
$$


Lemma 3 Any strong graph $G$ with parts $A$ and $B$ contains a critical subgraph $G^{*}$ with parts $A^{*} \subseteq A$ and $B^{*} \subseteq B$.

Proof: Let $E^{\prime}$ be the maximum set of l-edges in $G$ such that $\beta\left(G^{\prime}\right)<k$, where $V\left(G^{\prime}\right)=$ $V(G)$ and $E\left(G^{\prime}\right)=E(G)-E^{\prime}$. Since $E^{\prime}$ is maximum and deleting all l-edges from $G$ produces the graph with independence number $k$, we obtain $\beta\left(G^{\prime}-e\right)=k$ for any l-edge $e \in E\left(G^{\prime}\right)$. Suppose that $G^{\prime}$ contains a vertex $u$ of degree 1 , and denote by $u v$ the b-edge incident to $u$. Let us show that $\operatorname{deg}_{G^{\prime}} v=1$. Suppose to the contrary that there is an l-edge $v w$ in $G^{\prime}$. Since $\beta\left(G^{\prime}-v w\right)=k$, there is an independent set $I$ in $G^{\prime}-v w$ of cardinality $k$. We have $v, w \in I$, for otherwise $I$ is independent in $G^{\prime}$, contrary to the fact that $\beta\left(G^{\prime}\right)<k$. Now the set $I^{\prime}=(I-\{v\}) \cup\{u\}$ is independent in $G^{\prime}$ and $\left|I^{\prime}\right|=|I|=k$, a contradiction again. Consequently, $\operatorname{deg}_{G^{\prime}} v=1$. Thus, if $\operatorname{deg}_{G^{\prime}} u=1$, then the b-edge incident to $u$ is an isolated edge in $G^{\prime}$.

Consider now the connected component $G^{*}\left(A^{*}, B^{*}\right)$ of the graph $G^{\prime}$ such that $A^{*} \subseteq A$, $B^{*} \subseteq B$ and $\beta\left(G^{*}\right)<k^{*}$, where $k^{*}=\left|A^{*}\right|=\left|B^{*}\right|$. Such a component does exist, for otherwise $\beta(H)=\frac{1}{2}|H|$ for each connected component $H$ of $G^{\prime}$ and hence $\beta\left(G^{\prime}\right)=\frac{1}{2}\left|G^{\prime}\right|=$ $k$, a contradiction. We see that $G^{*}$ is a connected strong graph of order $2 k^{*}$. If $\delta\left(G^{*}\right)=1$, then $G^{*}$ is an isolated b-edge in $G^{\prime}$ and so $\beta\left(G^{*}\right)=k^{*}=1$, a contradiction. Hence $\delta\left(G^{*}\right) \geq 2$. If there exists an l-edge $e$ in $G^{*}$ such that $\beta\left(G^{*}-e\right)<k^{*}$, then obviously $\beta\left(G^{\prime}-e\right)<k$, contrary to the maximality of $E^{\prime}$. Thus, $\beta\left(G^{*}-e\right)=k^{*}$ for any l-edge $e \in E\left(G^{*}\right)$. We conclude that $G^{*}$ is a critical graph.

Lemma $4 A$ graph $G$ is $\Gamma$-perfect if and only if $G$ contains no critical graph as a semiinduced subgraph.

Proof: Let $G$ be a $\Gamma$-perfect graph and suppose that $G$ contains a critical graph $H(A, B)$ as a semi-induced subgraph. We have $\beta(H)<k$, where $k=|A|=|B|$. Consider in the graph $G$ the induced subgraph $F=\langle A \cup B\rangle$. This graph is obtained from $H$ by adding some edges in the parts $A, B$. Therefore, $\beta(F)<k$ and $F$ is a $\mathrm{W}$-graph, i.e., $F$ is a strong graph. This is a contradiction, since, by Lemma 2, the graph $G$ does not contain any strong graph as an induced subgraph.

Now let $G$ contain no critical graph as a semi-induced subgraph, and suppose that $G$ is not $\Gamma$-perfect. By Lemma 2, the graph $G$ contains a strong graph $H$ as an induced subgraph. Now, by Lemma $3, H(A, B)$ contains a critical subgraph $H^{*}\left(A^{*}, B^{*}\right)$ such that $A^{*} \subseteq A$ and $B^{*} \subseteq B$, i.e., $H^{*}$ is a semi-induced subgraph of $H$. Therefore, the critical graph $H^{*}$ is a semi-induced subgraph of $G$, a contradiction.

In the remaining part of the proof we give a description of the class of critical graphs. In fact we prove that a graph is critical if and only if it is a basis. This result together with Lemma 4 will provide the characterization of $\Gamma$-perfect graphs.

Lemma 5 If $G^{\prime}$ is obtained from a perfect bl-graph $G$ by the operation of $W$-reducibility, then $G^{\prime}$ is a $W$-graph whose perfect matching between the parts consists of b-edges determined by the operation of $W$-reducibility.

Proof: Let $G^{\prime}$ be obtained from a perfect bl-graph $G$ by the operation of W-reducibility. Note that the graph $G$ may have loops only if $G=P r_{1}$. In that case the loops (l-edges) of 
$G$ are replaced in accordance with Definition 11 by alternating even l-cycles. If $u \in V\left(G^{\prime}\right)$ is an old vertex, i.e. $u \in V(G)$, then $u$ is labeled by $c(u)$ in $G^{\prime}$. If $u \in V\left(G^{\prime}\right)$ is a new vertex, then $u$ is a non-end vertex of some chain $P_{e}$. We label all vertices from $V\left(G^{\prime}\right)-V(G)$ by the following inductive rule. If $e=u v$ is an edge of $G^{\prime}$ such that $u$ has a label but $v$ has no label yet, then we put:

- $c(v)=A$ if $c(u)=A$ and $e$ is an l-edge.

- $c(v)=B$ if $c(u)=A$ and $e$ is a b-edge.

- $c(v)=A$ if $c(u)=B$ and $e$ is a b-edge.

- $c(v)=B$ if $c(u)=B$ and $e$ is an l-edge.

Now, the vertices of $G^{\prime}$ with label $A$ form the part $A$, the vertices with label $B$ form the part $B$, and the set of b-edges of $G^{\prime}$ forms a perfect matching between $A$ and $B$, i.e., the sets $A, B$ independently match each other in $G^{\prime}$. Thus, the graph $G^{\prime}$ is a W-graph.

Lemma 6 Let $G$ be a perfect bl-graph of order $2 k$ and let $C=\left(u_{1}, u_{2}, \ldots, u_{2 n+1}\right)$ be an l-cycle in $G$ starting with $u_{1}$. If $\beta(G)=k$, then the vertex $u_{1}$ belongs to no maximum independent set of $G$.

Proof: By definition, the edge $u_{2 i} u_{2 i+1}$ is a b-edge for any $i, 1 \leq i \leq n$. Suppose that there is a maximum independent set $I$ containing $u_{1}$. Since $\beta(G)=k$, the set $I$ contains exactly one vertex of each b-edge. We have $u_{1} \in I$ and hence $u_{2} \notin I$. Therefore, $u_{3} \in I$. If we continue this process, we finally arrive at $u_{2 n+1} \in I$. This is a contradiction, since the set $I$ contains two adjacent vertices $u_{1}$ and $u_{2 n+1}$.

Definition 17 A perfect bl-graph $G$ is called a semi-basis if $G$ consists of two l-cycles $C$ and $C^{\prime}$ starting with $u$ and $u^{\prime}\left(u \neq u^{\prime}\right)$, respectively, and also of a b-chain $P$ connecting $u$ and $u^{\prime}$. Note that $C, C^{\prime}$ and $P$ do not necessarily contain different vertices. However, any of the graphs $C, C^{\prime}$ or $P$ has no self-intersections, since it is simple.

Lemma 7 If a perfect bl-graph $G$ of order $2 k$ contains a semi-basis subgraph, then

$$
\beta(G)<k \text {. }
$$

Proof: Suppose to the contrary that $G$ has an independent set $I$ of cardinality $k$. Then, obviously, $\beta(G)=k$. By Lemma 6 , the starting vertices $u, u^{\prime}$ of the l-cycles $C, C^{\prime}$ do not belong to the set $I$. Let $P=\left(u_{1}, u_{2}, \ldots, u_{2 m}\right)$ be a b-chain connecting $u=u_{1}$ and $u^{\prime}=u_{2 m}$. The edges $u_{2 i-1} u_{2 i}(1 \leq i \leq m)$ are b-edges and the set $I$ contains exactly one vertex of each b-edge, since $\beta(G)=k$. The vertex $u=u_{1}$ does not belong to $I$, and so $u_{2} \in I$. Hence $u_{3} \notin I$ and $u_{4} \in I$. Going on in the same way, we obtain $u_{2 i} \in I$ for all $i, 1 \leq i \leq m$. This is a contradiction, since the vertex $u^{\prime}=u_{2 m}$ does not belong to the set $I$.

Lemma 8 A critical graph $G$ is a semi-basis. The graph $G$-e does not contain a semibasis subgraph for any l-edge $e \in E(G)$. 
Proof: Let $v$ be an arbitrary vertex of a critical graph $G(A, B)$, say $v \in A$. Put $X_{0}=\{v\}$ and $X_{0}^{\prime}=N(v) \cap A$. For $i \geq 0$, we define the sets $X_{i+1}$ and $X_{i+1}^{\prime}$ as follows:

$$
\begin{gathered}
X_{i+1}=\left\{x \in V(G): x y \text { is a b-edge, } y \in X_{i}^{\prime}\right\}, \\
X_{i+1}^{\prime}=N\left(X_{i+1}\right)-\left(\cup_{j=0}^{i} X_{j}^{\prime} \cup\left\{v^{\prime}\right\}\right),
\end{gathered}
$$

where $v^{\prime} \in B$ and $v v^{\prime}$ is a b-edge. The construction of the sequence

$$
X_{0}, X_{0}^{\prime}, X_{1}, X_{1}^{\prime}, \ldots, X_{n}, X_{n}^{\prime}
$$

is finished for minimal $n$ such that $X_{n}^{\prime}=\emptyset$. Clearly, the above sets are pairwise disjoint. Put

$$
\begin{gathered}
X=\cup_{j=0}^{n} X_{j}, \\
X^{\prime}=\cup_{j=0}^{n-1} X_{j}^{\prime} \cup\left\{v^{\prime}\right\} .
\end{gathered}
$$

Let us show that the set $X$ is not independent. The graph $G$ is critical, and so $\delta(G) \geq 2$. Hence there is a vertex $w \in A$ adjacent to $v$. Moreover, $\beta(G-v w)=k=|A|=|B|$. Let $I$ be an independent set of $G-v w$ of cardinality $k$. Since $I$ is not independent in $G$, we have $v, w \in I$. Put

$$
\begin{aligned}
A_{1} & =A-\left(X \cup X^{\prime}\right), \\
B_{1} & =B-\left(X \cup X^{\prime}\right), \\
I_{1} & =A_{1} \cap I, \\
I_{2} & =B_{1} \cap I .
\end{aligned}
$$

By the definitions, no vertex of $X$ is adjacent to a vertex of $A_{1} \cup B_{1}$. The set $I^{\prime}=X \cup I_{1} \cup I_{2}$ has cardinality $k$, and hence $I^{\prime}$ is not independent in $G$. On the other hand, $I_{1} \cup I_{2}$ is independent in $G$ and there is no edge between $I_{1} \cup I_{2}$ and $X$ in $G$. We conclude that $X$ is not independent, and hence $x_{s} \in X_{s}$ is adjacent to $y_{t} \in X_{t}$. Clearly, $s$ and $t$ have the same parity. If $s<t$, then we have a contradiction, since $y_{t}$ must belong to $X_{s}^{\prime}$ but $X_{s}^{\prime} \cap X_{t}=\emptyset$. Thus, $s=t$, i.e., $x_{s} \in X_{s}$ is adjacent to $y_{s} \in X_{s}$. Now we construct two alternating simple chains. Put

$$
\begin{gathered}
P_{1}=\left(x_{s}, x_{s-1}^{\prime}, x_{s-1}, x_{s-2}^{\prime}, \ldots, x_{0}^{\prime}, x_{0}=v\right), \\
P_{2}=\left(y_{s}, y_{s-1}^{\prime}, y_{s-1}, y_{s-2}^{\prime}, \ldots, y_{0}^{\prime}, y_{0}=v\right),
\end{gathered}
$$

where $x_{i}, y_{i} \in X_{i}$ and $x_{i}^{\prime}, y_{i}^{\prime} \in X_{i}^{\prime}(0 \leq i \leq s)$. Let $z$ be the first common vertex of $P_{1}$ and $P_{2}$ if we go from $x_{s}$ to $v$ (possibly, $z=v$ ). Obviously, $z \in X$. Now, the edge $x_{s} y_{s}$, the $\left(x_{s}, z\right)$-subchain of $P_{1}$ and the $\left(y_{s}, z\right)$-subchain of $P_{2}$ form the l-cycle $C$ starting with $z$. If $z \neq v$, then the $(z, v)$-subchain of $P_{1}$ is an alternating $(z, v)$-chain in which $z$ is incident to a b-edge and $v$ is incident to an l-edge.

In fact we proved the following lemma.

Lemma 9 For any vertex $v$ of a critical graph $G$, there exists an l-cycle $C$ starting with $z$ and such that if $v \neq z$, then there is an alternating $(v, z)$-chain in which $v$ is incident to an l-edge, and $z$ is incident to a b-edge, and moreover, $z$ is the only common vertex of this chain and $C$. 
We go on with the proof of Lemma 8. Denote by $z z_{1}$ the b-edge incident to the vertex $z$, and apply Lemma 9 to the vertex $z_{1}$. Let $C^{\prime}$ be the l-cycle starting with $z^{\prime}$. If $z_{1} \neq z^{\prime}$, let $P$ be the alternating $\left(z^{\prime}, z_{1}\right)$-chain in which $z_{1}$ is incident to an l-edge and $z^{\prime}$ is incident to a b-edge. If $z_{1}=z^{\prime}$, then put $P=\emptyset$. Let $P^{+}=P \cup z_{1} z$, thus $P^{+}$is the b-chain connecting the starting vertices $z$ and $z^{\prime}$ of the l-cycles $C$ and $C^{\prime}$. The union of the cycles $C, C^{\prime}$ and the chain $P^{+}$produces a semi-basis subgraph $G^{\prime}$ of the graph $G$. The semi-basis graph $G^{\prime}$ is shown in Fig. 2 provided that it has no self-intersections.

Let $e$ be an l-edge of the graph $G$ and suppose that the graph $G-e$ contains a semibasis subgraph. By Lemma $7, \beta(G-e)<k$. On the other hand, $G$ is critical, and so $\beta(G-e)=k$, a contradiction. Thus, the graph $G-e$ does not contain a semi-basis subgraph for any l-edge $e \in E(G)$. Therefore, the semi-basis subgraph $G^{\prime}$ of $G$ contains all l-edges of $G$. Since $G$ is critical, we have $\delta(G) \geq 2$. Hence $V\left(G^{\prime}\right)=V(G)$. Taking into account that any semi-basis graph is a perfect bl-graph, we conclude that $G^{\prime}$ must contain all b-edges of $G$. Thus, $G^{\prime}=G$. The proof of Lemma 8 is complete.

Remark 1 The proof of Lemma 8 implies that the cycle $C^{\prime}$ and the chain $P-\left\{z_{1}\right\}$ may intersect the set $V(C)-\{z\}$ in the critical graph $G$, all other intersections are impossible.

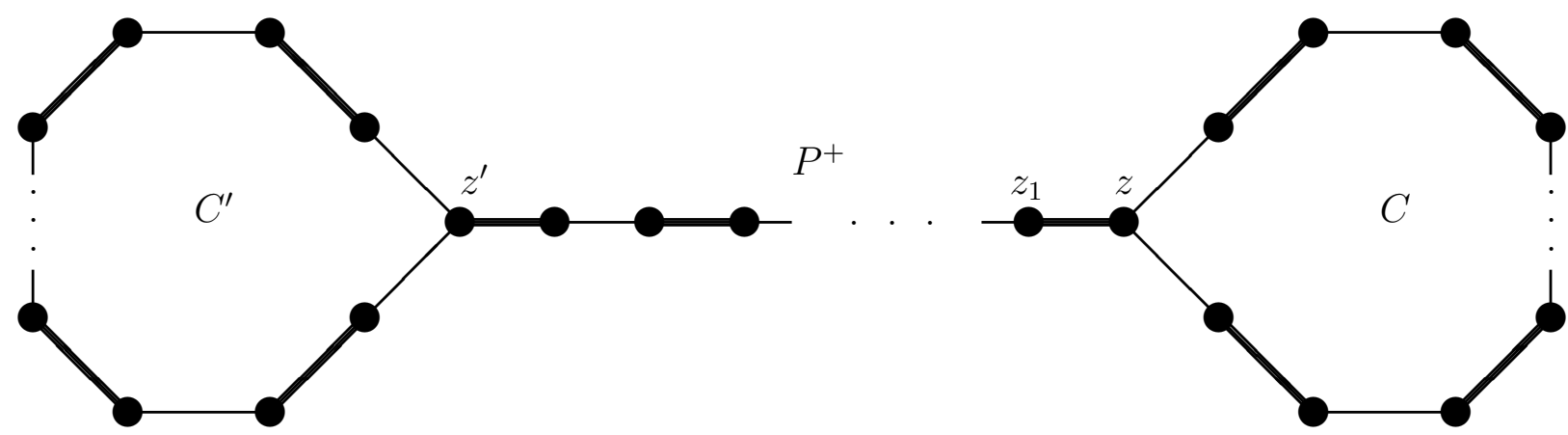

FIGURE 2. Semi-basis graph without self-intersections.

Lemma 10 Any critical graph is a basis.

Proof: Let $G$ be a critical graph. By Lemma 8, the graph $G$ is a semi-basis. Employing the notation used in Lemma 8 and taking into account Remark 1, we consider all possible intersections of $V\left(C^{\prime}\right) \cup\left(V(P)-\left\{z_{1}\right\}\right)$ and $V(C)-\{z\}$. Suppose that the intersection of these sets is empty. The graph $G$ is a W-graph, since $G$ is critical. Hence the cycles $C$ and $C^{\prime}$ have the even number of b-edges. Therefore, $G$ can be obtained from $P r_{1}$ by the operation of $\mathrm{W}$-reducibility, i.e., $G$ is a basis.

Suppose that $u$ is a common vertex of $C^{\prime} \cup P$ and $C$. Let $Q$ be a maximal common subchain of $C^{\prime} \cup P$ and $C$ such that $Q$ contains $u$. Since every vertex in $G$ is incident to exactly one b-edge, it follows that $Q$ is a b-chain. Maximal common b-chains will be called intersection intervals. Obviously, $\operatorname{deg}_{G} v=2$ for any non-end vertex $v$ of any intersection interval. 
Let us show that the set $V(P)-\left\{z_{1}\right\}$ does not intersect the set $V(C)-\{z\}$. Suppose to the contrary that $u \in\left(V(P)-\left\{z_{1}\right\}\right) \cap(V(C)-\{z\})$ and $u$ is the nearest vertex to the vertex $z$ in the chain $P^{+}$. Denote by $\left(u, u^{\prime}\right)$ the corresponding intersection interval. Let $L$ be the $(z, u)$-subchain of $C$ such that $u^{\prime} \in L$, and let $y \in L$ be adjacent to $z$. Thus, $z y$ is an l-edge. The $(z, u)$-subchain of $P^{+}$and the $(u, z)$-subchain of $C$ not containing $u^{\prime}$ form the l-cycle $C^{\prime \prime}$ starting with $u$. The $\left(u, z^{\prime}\right)$-subchain $P^{\prime}$ of the chain $P^{+}$connects $C^{\prime \prime}$ with the l-cycle $C^{\prime}$ starting with $z^{\prime}$. Thus, for the l-edge $z y$ the graph $G-z y$ contains a semi-basis subgraph formed by $C^{\prime}, C^{\prime \prime}$ and $P^{\prime}$, contrary to Lemma 8. Therefore, $V(P) \cap V(C)=\emptyset$.

Now consider possible intersections of $C^{\prime}-\left\{z^{\prime}\right\}$ and $C-\{z\}$. Passing round the cycle $C^{\prime}$ from the vertex $z^{\prime}$, denote all intersection intervals by

$$
\left(u_{1}, u_{1}^{\prime}\right),\left(u_{2}, u_{2}^{\prime}\right), \ldots,\left(u_{t}, u_{t}^{\prime}\right)
$$

where $u_{i}, u_{i}^{\prime}(1 \leq i \leq t)$ are end vertices of the intervals. Since $C^{\prime} \cap C \neq \emptyset$, we have $t \geq 1$. In what follows it is always supposed that we pass round the cycle $C$ in the direction from $u_{1}^{\prime}$ to $u_{1}$. We will prove that, passing round the cycle $C$ in this direction, the end vertices of the above intersection intervals are arranged in the following sequence:

$$
u_{1}^{\prime}, u_{1}, u_{2}^{\prime}, u_{2}, \ldots, u_{t}^{\prime}, u_{t}
$$

and moreover the vertex $z$ belongs to the $\left(u_{t}, u_{1}^{\prime}\right)$-subchain of $C$. These statements hold for $t=1$, and so we may assume that $t \geq 2$.

Suppose that passing round $C$ we arrive to the vertex $u_{1}^{\prime}$ from $u_{s}(s>1)$, i.e. the $\left(u_{s}, u_{1}^{\prime}\right)$-subchain $R$ of $C$ contains no end vertices of any intersection interval excepting $u_{s}$ and $u_{1}^{\prime}$, and the vertex $z$ does not belong to the chain $R$. We see that $R$ is an l-chain. Hence the chain $R$ and the $\left(u_{s}, u_{1}^{\prime}\right)$-subchain of $C^{\prime}$ containing $z^{\prime}$ form the l-cycle $C^{\prime \prime}$ starting with $z^{\prime}$. Let $e=v u_{1}^{\prime}$ be the l-edge of $C^{\prime}$. Obviously, $e \notin C^{\prime \prime}$ and $e \notin C$, since $\left(u_{1}^{\prime}, u_{1}\right)$ is a maximal common b-chain of $C$ and $C^{\prime}$. Thus, $G-e$ contains a semi-basis subgraph consisting of $C, C^{\prime \prime}$ and $P^{+}$, contrary to Lemma 8. Now suppose that passing round $C$ we arrive to $u_{1}^{\prime}$ from $u_{s}^{\prime}$, and $z$ does not belong to the $\left(u_{s}^{\prime}, u_{1}^{\prime}\right)$-subchain $R$ of $C$. Therefore, $R$ is an l-chain. The chain $R$ and the $\left(u_{1}^{\prime}, u_{s}^{\prime}\right)$-subchain of $C^{\prime}$ not containing $z^{\prime}$ form the l-cycle $C^{\prime \prime}$ starting with $u_{1}^{\prime}$. Let $e=v u_{s}^{\prime}$ be the l-edge of $C^{\prime}$. Obviously, $e \notin C^{\prime \prime}$ and $e \notin C$. Thus, $G-e$ contains a semi-basis subgraph consisting of $C, C^{\prime \prime}, P^{+}$and the $\left(z^{\prime}, u_{1}^{\prime}\right)$-subchain of $C^{\prime}$ not containing $u_{s}^{\prime}$, contrary to Lemma 8. Therefore, passing round $C$ we arrive to the vertex $u_{1}^{\prime}$ from $z$, and the $\left(z, u_{1}^{\prime}\right)$-subchain of $C$ contains no end vertex of any intersection interval excepting $u_{1}^{\prime}$.

Suppose now that passing round $C$ we arrive to the vertex $u_{r}$ and the next end vertex of the intersection intervals is $u_{s}$. It is evident that we arrived to the vertex $u_{r}$ from the vertex $u_{r}^{\prime}$. Since $u_{s} \neq u_{1}^{\prime}$, the $\left(u_{r}, u_{s}\right)$-subchain of $C$ does not contain $z$ and hence it is an l-chain. Let $s>r$. Let us define the cycle $C^{\prime \prime}$ consisting of the $\left(u_{r}, u_{s}\right)$-subchain of $C$ and the $\left(u_{r}, u_{s}\right)$-subchain of $C^{\prime}$ containing $u_{r}^{\prime}$. The cycle $C^{\prime \prime}$ is an l-cycle starting with $u_{s}$. Let $P^{\prime}$ be the $\left(u_{s}, z^{\prime}\right)$-subchain of $C^{\prime}$ containing $u_{s}^{\prime}$. Thus, $P^{\prime} \cup P^{+}$is a b-chain from $u_{s}$ to $z$. Let $e=z^{\prime} w$ be the l-edge of $C^{\prime}$ such that $e \notin P^{\prime}$. Since $z^{\prime} \notin C$, we have $e \notin C$. Also, $e \notin C^{\prime \prime}$. We conclude that the graph $G-e$ contains a semi-basis subgraph consisting of $C$, $C^{\prime \prime}$ and $P^{\prime} \cup P^{+}$, contrary to Lemma 8. Now let $s<r$. Let us define the cycle $C^{\prime \prime}$ consisting of the $\left(u_{r}, u_{s}\right)$-subchain of $C$ and the $\left(u_{s}, u_{r}\right)$-subchain of $C^{\prime}$ containing $u_{s}^{\prime}$. The cycle $C^{\prime \prime}$ is an l-cycle starting with $u_{r}$. Let $P^{\prime}$ be the $\left(u_{r}, z^{\prime}\right)$-subchain of $C^{\prime}$ containing $u_{r}^{\prime}$. Thus, 
$P^{\prime} \cup P^{+}$is a b-chain from $u_{r}$ to $z$. Let $e=z^{\prime} w$ be the l-edge of $C^{\prime}$ such that $e \notin P^{\prime}$. We have, $e \notin C$ and $e \notin C^{\prime \prime}$. We conclude that the graph $G-e$ contains a semi-basis subgraph consisting of $C, C^{\prime \prime}$ and $P^{\prime} \cup P^{+}$, contrary to Lemma 8. Thus, if we arrive to the vertex $u_{r}$ passing round $C$, then the next end vertex of the intersection intervals must be $u_{s}^{\prime}$, i.e., passing round the cycle $C$ the end vertices of the intersection intervals are arranged in the following sequence:

$$
u_{1}^{\prime}, u_{1}, u_{f(2)}^{\prime}, u_{f(2)}, \ldots, u_{f(t)}^{\prime}, u_{f(t)},
$$

where $f:\{2,3, . ., t\} \rightarrow\{2,3, \ldots, t\}$ is a bijection. Clearly, $z$ belongs to the $\left(u_{f(t)}, u_{1}^{\prime}\right)$ subchain of $C$. Assume that there is $j \in\{2,3, \ldots, t\}$ such that $f(j-1)>f(j)$. Denote $r=f(j-1)$ and $s=f(j)$. Thus, $r>s$. Let $L$ be the $\left(u_{r}, u_{s}^{\prime}\right)$-subchain of $C$. We see that $z \notin L$ and hence $L$ is an l-chain. Let $L^{\prime}$ be the $\left(u_{s}^{\prime}, u_{r}\right)$-subchain of $C^{\prime}$ not containing $z^{\prime}$. Obviously, $L^{\prime}$ is an l-chain. Replace the chain $L^{\prime}$ in $C^{\prime}$ by the chain $L$ and denote the resulting cycle by $C^{\prime \prime}$. The cycle $C^{\prime \prime}$ is an l-cycle starting with $z^{\prime}$. Let $e=u_{r} w$ be the l-edge of $L^{\prime}$. It is evident that $e \notin C^{\prime \prime}$ and $e \notin C$. We deduce that the graph $G-e$ contains a semi-basis subgraph consisting of $C, C^{\prime \prime}$ and $P^{+}$, contrary to Lemma 8. Consequently, the end vertices of intersection intervals while passing round $C$ are arranged in accordance with (1).

The graph $G(A, B)$ is a W-graph, since $G$ is critical. We label all vertices of $G$ as follows. Put $c(v)=A$ if $v \in A$, and $c(v)=B$ if $v \in B$. Denote $u_{0}=z$ and $u_{0}^{\prime}=z^{\prime}$. Furthermore, we construct the graph $G^{*}$ by the following rule. Let

$$
\mathcal{P}_{b}=\left\{\left(u_{i}, u_{i}^{\prime}\right): 0 \leq i \leq t\right\}
$$

be the set of b-chains of $G$ consisting of the chain $P^{+}$and the intersection intervals. Replace each chain $\left(u_{i}, u_{i}^{\prime}\right)$ from $\mathcal{P}_{b}$ by the b-edge $u_{i} u_{i}^{\prime}$. Note that the chain $\left(u_{i}, u_{i}^{\prime}\right)$ is an even bchain if $u_{i}$ and $u_{i}^{\prime}$ have the same label, and this chain is an odd b-chain otherwise. Now let

$$
\mathcal{P}_{l}=\left\{\left(u_{t}, u_{0}\right),\left(u_{t}^{\prime}, u_{0}^{\prime}\right),\left(u_{i-1}, u_{i}^{\prime}\right),\left(u_{i-1}^{\prime}, u_{i}\right): 1 \leq i \leq t\right\}
$$

be the set of l-chains of $G$. Replace each chain $L$ from $\mathcal{P}_{l}$ by the l-edge connecting the end vertices of $L$. Note that $L$ is an even l-chain if its end vertices have the same label, and $L$ is an odd l-chain otherwise. The resulting graph $G^{*}$ is the odd prism $\operatorname{Pr}_{t+1}$ whenever $t \geq 1$ is even, and $G^{*}$ is the even Möbius ladder $M l_{t+1}$ whenever $t \geq 1$ is odd. Moreover, using the mapping $c: V\left(G^{*}\right) \rightarrow\{A, B\}$ constructed above, the graph $G$ is obtained from $G^{*}$ by the operation of W-reducibility. Therefore, $G$ is a basis graph. The proof of Lemma 10 is complete.

Lemma 11 All basis graphs are critical.

Proof: Let $G$ be a basis graph, i.e., $G$ is obtained from $\operatorname{Pr}_{2 n+1}(n \geq 0)$ or $M l_{2 n}(n \geq 1)$ by the operation of W-reducibility. By Lemma $5, G$ is a W-graph, $G=G(A, B)$. Obviously, $\delta(G) \geq 2$ and $G$ is a connected graph. Let us show that $\beta(G)<k$. By Lemma 7 , it is sufficient to find a semi-basis subgraph in $G$. If $G$ is obtained from $\operatorname{Pr}_{1}$, then $G$ is evidently a semi-basis subgraph. Now let $G$ be obtained from $P r_{2 n+1}$ or $M l_{2 n}, n \geq 1$. Since the operation of W-reducibility preserves semi-basis subgraphs, it is sufficient to find such a graph in $\operatorname{Pr}_{2 n+1}$ or $M l_{2 n}, n \geq 1$. In fact we will show that both $\operatorname{Pr}_{2 n+1}$ and $M l_{2 n}$ are semi-basis graphs. 
Recall that in $\operatorname{Pr}_{2 n+1}$, the cycles $\left(u_{1}, u_{2}, \ldots, u_{2 n+1}\right)$ and $\left(v_{1}, v_{2}, \ldots, v_{2 n+1}\right)$ consist of 1 edges, and $\left\{u_{i} v_{i}: 1 \leq i \leq 2 n+1\right\}$ is the set of b-edges. Define two cycles as follows:

$$
C=\left(u_{1}, u_{2}, v_{2}, v_{3}, u_{3}, u_{4}, \ldots, u_{2 n}, v_{2 n}, v_{2 n+1}, u_{2 n+1}, u_{1}\right)
$$

and

$$
C^{\prime}=\left(v_{1}, v_{2}, u_{2}, u_{3}, v_{3}, v_{4}, \ldots, v_{2 n}, u_{2 n}, u_{2 n+1}, v_{2 n+1}, v_{1}\right) .
$$

The cycle $C$ starting with $u_{1}$ and the cycle $C^{\prime}$ starting with $v_{1}$ are l-cycles connected by the b-chain $\left(u_{1}, v_{1}\right)=u_{1} v_{1}$, i.e., $P r_{2 n+1}$ is a semi-basis graph for $n \geq 1$.

Consider now the Möbius ladder $M l_{2 n}$. Recall that the cycle $\left(u_{1}, u_{2}, \ldots, u_{4 n}\right)$ in $M l_{2 n}$ consists of l-edges, and $\left\{u_{i}, u_{2 n+i}: 1 \leq i \leq 2 n\right\}$ is the set of b-edges of $M l_{2 n}$. Define two cycles as follows:

$$
C=\left(u_{1}, u_{2}, u_{2 n+2}, u_{2 n+3}, u_{3}, u_{4}, u_{2 n+4}, u_{2 n+5}, \ldots, u_{2 n}, u_{4 n}, u_{1}\right)
$$

and

$$
C^{\prime}=\left(u_{2 n+1}, u_{2 n+2}, u_{2}, u_{3}, u_{2 n+3}, u_{2 n+4}, u_{4}, u_{5}, \ldots, u_{4 n}, u_{2 n}, u_{2 n+1}\right) .
$$

The cycle $C$ starting with $u_{1}$ and the cycle $C^{\prime}$ starting with $u_{2 n+1}$ are l-cycles connected by the b-chain $\left(u_{1}, u_{2 n+1}\right)=u_{1} u_{2 n+1}$, i.e., $M l_{2 n}$ is a semi-basis graph.

Thus, $\beta(G)<k$. It remains to prove that $\beta(G-e)=k$ for each l-edge $e \in E(G)$. Let $G$ be obtained from $\operatorname{Pr}_{1}$ by the operation of W-reducibility. Obviously for any l-edge $e \in E(G)$, the graph $G-e$ contains 1 or 2 vertices of degree 1. Starting with a vertex (vertices) of degree 1 , it is easily to construct the desired independent set of cardinality $k$.

Now let $G$ be obtained from $H=\left\{P r_{2 n+1}, M l_{2 n}: n \geq 1\right\}$ by the operation of $\mathrm{W}$ reducibility, i.e., b-edges are replaced by alternating b-chains and l-edges are replaced by alternating l-chains. There are two cases to consider.

Case 1. The l-edge $e$ belongs to an l-chain $P_{f}$, where $f$ is an l-edge of $H$. If $H=P r_{2 n+1}$, then without loss of generality we may suppose that $f=u_{1} u_{2 n+1}$. Put

$$
I=\left\{u_{1}, u_{2 i+1}, v_{2 i}: 1 \leq i \leq n\right\} .
$$

If $H=M l_{2 n}$, then we may assume that $f=u_{1} u_{2}$. In that case put

$$
I=\left\{u_{1}, u_{2 i}, u_{2 n}, u_{2 n+2 i+1}: 1 \leq i \leq n-1\right\} .
$$

The set $I$ is an independent set of $H-f$ of cardinality $\frac{1}{2}|H|$. Now it is not difficult to construct an independent set of $G-e$ of cardinality $k=\frac{1}{2}|G|$. Indeed, let $u v \in E(H)$ be a b-edge replaced by a b-chain $P$. Since $|I|=\frac{1}{2}|H|$, we have $|\{u, v\} \cap I|=1$, say $u \in I$. From each b-edge of $P$ we add in $I$ one vertex which is nearer to the vertex $u$ in the chain $P$. If $u v \neq f$ is an l-edge of $H$ replaced by an l-chain $P$, then $|\{u, v\} \cap I| \leq 1$ and we can add vertices in $I$ in the same way as above. Now suppose that $u v=f$, and let $e=x y$. Then, from each b-edge of $P_{f}-e$ we add in $I$ one vertex which is nearer to the vertices $x, y$ in the chain $P_{f}$. The constructed set $I^{\prime}$ is an independent set of $G-e$. Since $I^{\prime}$ contains one vertex of each b-edge in $G-e$, we have $\left|I^{\prime}\right|=k$. Consequently, $\beta(G-e)=k$.

Case 2. The l-edge $e$ belongs to a b-chain $P_{f}$, where $f$ is a b-edge of $H$. If $H=P r_{2 n+1}$, then without loss of generality we may suppose that $f=u_{1} v_{1}$. Put

$$
I=\left\{u_{2 i}, v_{2 i+1}: 1 \leq i \leq n\right\} .
$$


If $H=M l_{2 n}$, then we may assume that $f=u_{1} u_{2 n+1}$. In that case put

$$
I=\left\{u_{2 i}, u_{2 n}, u_{2 n+2 i+1}: 1 \leq i \leq n-1\right\} .
$$

The set $I$ is an independent set of $H-f$ such that each b-edge of $H-f$ has one vertex in $I$ and $|I|=\frac{1}{2}|H|-1$. Note also that the end vertices of $f$ do not belong to $I$. Adding vertices in the set $I$ in the same way as in Case 1, we obtain the set $I^{\prime}$ such that $I^{\prime}$ contains one vertex of each b-edge of the graph $G-e$. Therefore, $\left|I^{\prime}\right|=k=\frac{1}{2}|G|$, i.e., $\beta(G-e)=k$.

Thus, Lemmas 10 and 11 imply that a graph is critical if and only if it is a basis. Now the proof of Theorem 1 follows from Lemma 4.

\section{Corollaries}

In this section we illustrate some applications of the characterization of $\Gamma$-perfect graphs in terms of forbidden semi-induced subgraphs. We say that a graph $G$ is 2-homeomorphic to $H$ if $G$ can be obtained from $H$ by replacing edges of $H$ by chains of even order $2 k, k \geq 1$. Let the family $\mathcal{H}$ consist of graphs 2-homeomorphic to the odd prism $\operatorname{Pr}_{2 n+1}(n \geq 0)$ or the even Möbius ladder $M l_{2 m}(m \geq 1)$.

Proposition 1 If $H$ belongs to $\mathcal{H}$, then $\beta(H)<\frac{1}{2}|H|$.

Proof: For the odd prism we have $\beta\left(P r_{2 n+1}\right)=2 n$, i.e., $\beta\left(P r_{2 n+1}\right)<\frac{1}{2}\left|P r_{2 n+1}\right|$. For the even Möbius ladder we have $\beta\left(M l_{2 m}\right)=2 m-1$, i.e., $\beta\left(M l_{2 m}\right)<\frac{1}{2}\left|M l_{2 m}\right|$. Let $F^{\prime}$ be obtained from a graph $F$ by the single 2-partition of the edge $u v$, i.e., $u v$ is replaced by the chain $P=(u, x, y, v)$. Let $U$ be a maximum independent set of $F^{\prime}$. Obviously, $1 \leq|U \cap P| \leq 2$. If $|U \cap P|=1$, then $U-P$ is an independent set of $F$ of cardinality $|U|-1=\beta\left(F^{\prime}\right)-1$. If $|U \cap P|=2$, then at least one vertex from $\{u, v\}$ belongs to $U$, say $u \in U$. Now $U-\{x, y, v\}$ is an independent set of $F$ of cardinality $|U|-1=\beta\left(F^{\prime}\right)-1$. In any case, $\beta(F) \geq \beta\left(F^{\prime}\right)-1$. Thus, if $\beta(F)<\frac{1}{2}|F|$, then

$$
\beta\left(F^{\prime}\right) \leq \beta(F)+1<\frac{1}{2}|F|+1=\frac{1}{2}\left|F^{\prime}\right| .
$$

Since $H$ is obtained from the odd prism or the even Möbius ladder by applying the operation of 2-partition, we conclude that $\beta(H)<\frac{1}{2}|H|$.

In our next theorem, the graphs from the family $\mathcal{H}$ are forbidden as semi-induced subgraphs for a graph to be $\Gamma$-perfect. Using the fact that a semi-induced subgraph of a graph is a $\mathrm{W}$-graph, we see that the class of forbidden semi-induced subgraphs of Theorem 2 actually consists of $\mathrm{W}$-graphs from the family $\mathcal{H}$. Note that the class of $\mathrm{W}$-graphs from $\mathcal{H}$ is larger than the class of basis graphs used in Theorem 1. For example, $M l_{4}=$ $M_{4}\left(\left\{u_{2}, u_{3}, u_{6}, u_{7}\right\},\left\{u_{1}, u_{4}, u_{5}, u_{8}\right\}\right)$ is a W-graph from $\mathcal{H}$ and hence it is forbidden in Theorem 2. On the other hand, $M l_{4}$ is not a basis graph. Another difference between Theorem 1 and Theorem 2 is that a basis graph has a fixed partition into parts determined by the set of its b-edges, while for a $\mathrm{W}$-graph from $\mathcal{H}$ the partition into parts is not fixed. 
Theorem 2 A graph $G$ is $\Gamma$-perfect if and only if $G$ does not contain a semi-induced subgraph 2-homeomorphic to the odd prism $\operatorname{Pr}_{2 n+1}(n \geq 0)$ or the even Möbius ladder $M l_{2 m}(m \geq 1)$.

Proof: Let $G$ be a $\Gamma$-perfect graph and let $H$ belong to $\mathcal{H}$. If $H$ is not a W-graph, then $H$ cannot be a semi-induced subgraph of $G$. Suppose now that $H=H(A, B)$ is a W-graph and $H$ is a semi-induced subgraph of $G$. By Proposition $1, \beta(H)<\frac{1}{2}|H|$. Let $H^{\prime}=\langle A \cup B\rangle$. Evidently, $\beta\left(H^{\prime}\right) \leq \beta(H)$ and $H^{\prime}$ is a $\mathrm{W}$-graph. Therefore, by Lemma 1 ,

$$
\Gamma\left(H^{\prime}\right)=\frac{1}{2}\left|H^{\prime}\right|=\frac{1}{2}|H|>\beta(H) \geq \beta\left(H^{\prime}\right) .
$$

Thus, $\Gamma\left(H^{\prime}\right)>\beta\left(H^{\prime}\right)$. This is a contradiction, since $G$ is a $\Gamma$-perfect graph.

Suppose that $G$ does not contain any graph from $\mathcal{H}$ as a semi-induced subgraph. Any basis graph $F$ is obtained from the odd prism or the even Möbius ladder by replacing its edges by alternating chains of even order, and the partition into parts of $F$ is determined by the set of its b-edges. Thus, the graph $F$ is 2-homeomorphic to the odd prism or the even Möbius ladder and $F$ has a fixed partition into parts. For a $\mathrm{W}$-graph $H$ from $\mathcal{H}$, the partition into parts of $H$ is not fixed, and hence we may take any partition $V(H)=A \cup B$ such that $A$ and $B$ independently match each other. Therefore, $F \in \mathcal{H}$ and the graph $G$ does not contain any basis graph as a semi-induced subgraph. The result now follows from Theorem 1.

Jacobson and Peters [22] considered the class of graphs $G$ having $\beta(H)=I R(H)$ for all induced subgraphs $H$ of $G$. By Theorem A, this class is exactly the class of $\Gamma$-perfect graphs.

Corollary 1 (Jacobson and Peters [22]) A graph $G$ is $\Gamma$-perfect if and only if for any vertex subsets $A, B \subset V(G)$ that independently match each other, the graph $\langle A \cup B\rangle$ has an independent set of order $|A|$.

Proof: Let $G$ be a $\Gamma$-perfect graph and $A, B$ independently match each other. The set $A$ is minimal dominating in $F=\langle A \cup B\rangle$. Hence, $\beta(F)=\Gamma(F) \geq|A|$. To prove the sufficiency, suppose that $G$ is not $\Gamma$-perfect. By Theorem 2, $G$ contains a semi-induced subgraph $H=H(A, B) \in \mathcal{H}$. By Proposition $1, \beta(H)<|A|$. Thus, the sets $A, B$ independently match each other in $G$ and $\beta\langle A \cup B\rangle<|A|$, a contradiction.

Now we turn to the problem of characterizing $\Gamma$-perfect graphs in terms of forbidden induced subgraphs. A graph $G$ is called minimal $\Gamma$-imperfect if $G$ is not $\Gamma$-perfect and $\beta(H)=\Gamma(H)$, for every proper induced subgraph $H$ of $G$.

Proposition 2 If $G$ is a minimal $\Gamma$-imperfect graph, then $G$ contains a basis graph $F(A, B)$ of order $2 k$ as a semi-induced subgraph, $G=G(A, B)$ is a connected W-graph of order $2 k$, $\delta(G) \geq 2$, and $\beta(G)=k-1$.

Proof: By Theorem 1, $G$ contains a basis graph $F$ as a semi-induced subgraph. Since $G$ is minimal, we have $V(G)=V(F)$. By Lemma 11, $F$ is critical, i.e., $F=F(A, B)$ is a connected W-graph of order $2 k, \delta(F) \geq 2$ and $\beta(F)<k$. The graph $G$ is obtained from $F$ by adding edges in the parts $A, B$. Therefore, $G(A, B)$ is a connected W-graph of order $2 k$, 
$\delta(G) \geq 2$ and $\beta(G)<k$. Let $u v$ be a b-edge of $G$. The graph $G^{\prime}=G-\{u, v\}$ is $\Gamma$-perfect. Hence, using Lemma $1, \beta\left(G^{\prime}\right)=\Gamma\left(G^{\prime}\right)=k-1$. We obtain $\beta(G) \geq \beta\left(G^{\prime}\right)=k-1$. Thus, $\beta(G)=k-1$.

By Proposition 2, every minimal $\Gamma$-imperfect graph has even order $n \geq 6$. Let $\mu_{n}$ denote the number of nonisomorphic minimal $\Gamma$-imperfect graphs of order $n$. It was proved in [14] that $\mu_{6}=1$ and $\mu_{8}=14$. Using a computer search, we discovered that $\mu_{10}=228$ and the number $\mu_{12}$ considerably exceeds $\mu_{10}$. Therefore, it seems unlikely to obtain an explicit list of all minimal $\Gamma$-imperfect graphs, i.e., to provide an induced subgraph characterization of the entire class of $\Gamma$-perfect graphs. However, for $K_{1,3}$-free $\Gamma$-perfect graphs Theorem 1 enables us to obtain such a characterization.

We define the family $\mathcal{S}$ consisting of the following classes $\mathcal{S}_{1}, \mathcal{S}_{2}$ and $\mathcal{S}_{3}$. Let $C=$ $C_{4 m}$ and $C^{\prime}=C_{4 n}(m, n \geq 1)$ be two cycles, let $u v \in E(C)$ and $x y \in E\left(C^{\prime}\right)$, and let $\left(z_{1}, z_{2}, \ldots, z_{2 l}\right)(l \geq 1)$ be a chain. Add the edges $u z_{1}, v z_{1}$ and $x z_{2 l}, y z_{2 l}$. The resulting graph belongs to $\mathcal{S}_{1}$. Now let $\left(u_{1}, \ldots, u_{k}\right),\left(v_{1}, \ldots, v_{l}\right)$ and $\left(w_{1}, \ldots, w_{m}\right)$ be three chains such that $k, l, m \geq 2$ and either $k, l, m \equiv 0(\bmod 4)$ or $k, l, m \equiv 2(\bmod 4)$. Adding the edges $u_{1} v_{1}$, $v_{1} w_{1}, w_{1} u_{1}$ and $u_{k} v_{l}, v_{l} w_{m}, w_{m} u_{k}$, we obtain a graph of the class $\mathcal{S}_{2}$. Lastly, let $C=C_{4 m}$ and $C^{\prime}=C_{4 n}(m, n \geq 1)$ be two cycles and let $u v \in E(C)$ and $x y \in E\left(C^{\prime}\right)$. Add the edges $u x, u y, v x, v y$. The resulting graph belongs to $\mathcal{S}_{3}$.

Theorem $3 A K_{1,3}$-free graph $G$ is $\Gamma$-perfect if and only if $G$ does not contain any member of $\mathcal{S}$ as an induced subgraph.

Proof: Any graph $H$ from the family $\mathcal{S}$ contains a semi-induced subgraph 2-homeomorphic to $\mathrm{Pr}_{1}, \mathrm{Pr}_{3}$ or $\mathrm{Ml}_{2}$. By Theorem 2, $\mathrm{H}$ is not $\Gamma$-perfect. To prove the sufficiency, let $G$ be a minimal counterexample, i.e., $G$ is a $K_{1,3}$-free graph not containing any member of $\mathcal{S}$ as an induced subgraph, $G$ is not $\Gamma$-perfect and $G$ has minimal order. Obviously, $G$ is a minimal $\Gamma$-imperfect graph. By Proposition 2, $G$ contains a basis graph $F=F(A, B)$ as a semi-induced subgraph, $G=G(A, B)$ is a connected W-graph of order $2 k, \delta(G) \geq 2$, and $\beta(G)=k-1$. If the induced subgraph $\langle A\rangle$ or $\langle B\rangle$ of the graph $G$ contains the induced chain $P_{3}$, then $G$ has the induced $K_{1,3}$, a contradiction. Hence both $\langle A\rangle$ and $\langle B\rangle$ are disjoint unions of complete graphs.

Lemma 12 Let $G(A, B)$ be a minimal $\Gamma$-imperfect graph of order $2 k$. If $\langle A\rangle$ and $\langle B\rangle$ are disjoint unions of complete graphs, then the following statements hold:

1. If $k$ is odd, then $\langle A\rangle \cong\langle B\rangle \cong \frac{k-3}{2} K_{2} \cup K_{3}(k \geq 3)$.

2. If $k$ is even, then one of the graphs $\langle A\rangle,\langle B\rangle$ is $\frac{k}{2} K_{2}$ and the other is either $\frac{k-4}{2} K_{2} \cup K_{4}$ $(k \geq 4)$ or $\frac{k-6}{2} K_{2} \cup 2 K_{3}(k \geq 6)$.

Proof: Let $\langle A\rangle$ be a disjoint union of the complete graphs $H_{1}, \ldots, H_{p}$. Since $\delta(G) \geq 2$, we have $\left|H_{i}\right| \geq 2$ for any $i \in\{1, \ldots, p\}$, and hence $p \leq k / 2$. Let $I$ be an independent set in $G$ of cardinality $k-1=\beta(G)$. Put $I_{A}=I \cap A$ and $I_{B}=I \cap B$. The set $I$ contains at most one vertex of each $H_{i}$, and hence $\left|I_{A}\right| \leq p \leq k / 2$. Analogously, $\left|I_{B}\right| \leq k / 2$. Further, $\left|I_{A}\right|=|I|-\left|I_{B}\right| \geq k-1-k / 2=k / 2-1$. Thus,

$$
k / 2-1 \leq\left|I_{A}\right| \leq k / 2
$$


Analogously,

$$
k / 2-1 \leq\left|I_{B}\right| \leq k / 2
$$

Put

$$
s=\sum_{i=1}^{p}\left(\left|H_{i}\right|-2\right) \geq 0
$$

We have,

$$
k=|A|=\sum_{i=1}^{p}\left|H_{i}\right|=s+2 p \geq s+2\left|I_{A}\right|
$$

Therefore, using (2),

$$
s \leq k-2\left|I_{A}\right| \leq 2 \text {. }
$$

Thus,

$$
s \in\{0,1,2\} .
$$

If $k=|A|$ is odd, then $s$ is also odd, since $s=k-2 p$. Hence $s=1, k \geq 3$, and $\langle A\rangle \cong \frac{k-3}{2} K_{2} \cup K_{3}$. Analogously, $\langle B\rangle \cong \frac{k-3}{2} K_{2} \cup K_{3}$.

Now let $k$ be even. Using (2) and (3), we see that one of the sets $I_{A}$ and $I_{B}$ has cardinality $k / 2-1$ and the other has cardinality $k / 2$. Without loss of generality, let $\left|I_{A}\right|=k / 2-1$ and $\left|I_{B}\right|=k / 2$. Since $\langle B\rangle$ is a disjoint union of complete graphs and $\delta(G) \geq 2$, we have $\langle B\rangle \cong \frac{k}{2} K_{2}$. Further, $s=k-2 p$ and $k$ is even. Hence $s$ is even and $s=0$ or 2 . If $s=0$, then $\langle A\rangle \cong \frac{k}{2} K_{2}$ and therefore $G$ is a disjoint union of even simple cycles. We obtain $\beta(G)=k$, a contradiction. Thus, $s=2$. Hence $k \geq 4$ and $\langle A\rangle \cong \frac{k-4}{2} K_{2} \cup K_{4}$ or $k \geq 6$ and $\langle A\rangle \cong \frac{k-6}{2} K_{2} \cup 2 K_{3}$. The proof of Lemma 12 is complete.

By Lemma 12, $G$ has either exactly 6 vertices of degree 3 or exactly 4 vertices of degree 4 , and all other vertices have degree 2. The basis graph $F$ is a spanning subgraph of $G$. Therefore, either $F$ has at most 6 vertices of degree 3 and all other vertices have degree 2 , or $F$ has at most 4 vertices of degree 3 and 4 and all other vertices have degree 2. Consequently, $F$ is obtained from $\mathrm{Pr}_{1}, \mathrm{Pr}_{3}$ or $\mathrm{Ml}_{2}$ (see Fig. 1) by the operation of W-reducibility. By Lemma 5, any l-edge of $F$ belongs to $A$ or $B$.

Suppose that $F$ is obtained from $P r_{1}$. Let $u u_{1}, u_{1} u^{\prime}$ be l-edges of one l-cycle of $F$ and let $v v_{1}, v_{1} v^{\prime}$ be l-edges of the other l-cycle of $F$. The vertices $u, u^{\prime}, u_{1}$ belong to the same part, and $v, v^{\prime}, v_{1}$ belong to the same part. We have, $u u^{\prime} \in E(G)$ and $v v^{\prime} \in E(G)$, since $G$ is a $K_{1,3}$-free graph. The restrictions on the degrees of vertices of $G$ imply $G=F \cup\left\{u u^{\prime}, v v^{\prime}\right\}$. Since $G$ is a W-graph, we see that the cycle $C$ of $G$ such that $u, u^{\prime} \in C$ and $u_{1} \notin C$ has length $4 m$, and the cycle $C^{\prime}$ of $G$ such that $v, v^{\prime} \in C^{\prime}$ and $v_{1} \notin C^{\prime}$ has length $4 n$. Thus, $G \in \mathcal{S}_{1}$, a contradiction.

Assume that $F$ is obtained from $\mathrm{Pr}_{3}$. The prism $\operatorname{Pr}_{3}$ has 6 vertices of degree 3. Hence, $G=F$. Suppose that an l-edge of $\operatorname{Pr}_{3}$ was replaced by an l-chain having more than 2 vertices. Then $F$ has the induced $K_{1,3}$, a contradiction. Therefore, only b-edges of $\mathrm{Pr}_{3}$ could be replaced by b-chains to obtain $F$. These chains must be odd b-chains if $C_{1}=\left(u_{1}, u_{2}, u_{3}\right)$ and $C_{2}=\left(v_{1}, v_{2}, v_{3}\right)$ belong to different parts of $F$, and they must be even b-chains if $C_{1}$ and $C_{2}$ belong to the same part of $F$. Any odd b-chain has $4 k+2$ vertices, and any even b-chain has $4 m$ vertices. Therefore, $G=F \in \mathcal{S}_{2}$, a contradiction. 
Finally, suppose that $F$ is obtained from $M l_{2}$ by the operation of $\mathrm{W}$-reducibility. It is easy to see that $M l_{2}$ has 4 different labelings of $V\left(M l_{2}\right)$ by $c(u) \in\{A, B\}$ up to replacing $A$ by $B$. Hence there are 4 cases to consider.

Case 1: $c\left(u_{1}\right)=c\left(u_{4}\right)=A$ and $c\left(u_{2}\right)=c\left(u_{3}\right)=B$. By the definition of W-reducibility, the l-edges $u_{1} u_{2}$ and $u_{3} u_{4}$ had to be replaced by the odd l-chains $\left(u_{1}, v_{1}, \ldots, v_{k}, u_{2}\right), k \geq 2$, and $\left(u_{3}, w_{1}, \ldots, w_{m}, u_{4}\right), m \geq 2$. Each of the vertices $v_{1}, v_{k}, w_{1}, w_{m}$ is an end vertex of $P_{3}$ or $P_{4}$ consisting of l-edges and hence belonging to $A$ or $B$. Since any part of $G$ is a disjoint union of complete graphs, we see that each of the above vertices will have degree at least 3 in $G$. Thus, $G$ has at least 8 vertices of degree at least 3, a contradiction.

Case 2: $c\left(u_{1}\right)=c\left(u_{3}\right)=A$ and $c\left(u_{2}\right)=c\left(u_{4}\right)=B$. This case is analogous to Case 1 , since the l-edges $u_{1} u_{2}$ and $u_{3} u_{4}$ had to be replaced by odd l-chains.

Case 3: $c\left(u_{1}\right)=c\left(u_{2}\right)=c\left(u_{3}\right)=A$ and $c\left(u_{4}\right)=B$. The l-edges $u_{1} u_{4}$ and $u_{3} u_{4}$ had to be replaced by the odd l-chains $\left(u_{1}, v_{1}, \ldots, v_{k}, u_{4}\right), k \geq 2$, and $\left(u_{3}, w_{1}, . ., w_{m}, u_{4}\right), m \geq 2$. Each of the vertices $v_{1}, v_{k}, w_{1}, w_{m}$ is an end vertex of $P_{r}(r \geq 3)$ consisting of l-edges. Hence, each of these vertices has degree at least 3 in $G$. Thus, $G$ has at least 8 vertices of degree at least 3 , a contradiction.

Case 4: $c\left(u_{i}\right)=A, 1 \leq i \leq 4$. If some two l-edges from $\left\{u_{1} u_{2}, u_{2} u_{3}, u_{3} u_{4}, u_{4} u_{1}\right\}$ were replaced by even l-chains having at least two b-edges, then we derive a contradiction in the same way as above. Suppose that only one l-edge, say $u_{1} u_{2}$, was replaced by the even l-chain $\left(u_{1}, v_{1}, \ldots, v_{k}, u_{2}\right), k \geq 4$. Then $\left\langle v_{k}, u_{2}, u_{3}, u_{4}, u_{1}, v_{1}\right\rangle$ is a $P_{6}$ in $F$ consisting of l-edges. Therefore, $G$ contains $K_{6}$, a contradiction. Thus, only the b-edges $u_{1} u_{3}$ and $u_{2} u_{4}$ of $M l_{2}$ were replaced by even b-chains and $\left\langle u_{1}, u_{2}, u_{3}, u_{4}\right\rangle$ is a $C_{4}$ in $F$ consisting of l-edges. Hence $\left\langle u_{1}, u_{2}, u_{3}, u_{4}\right\rangle$ is a $K_{4}$ in $G$, and so all other vertices in $G$ must have degree 2, i.e., $G=F \cup\left\{u_{1} u_{3}, u_{2} u_{4}\right\}$. Since even b-chains have $4 m$ vertices $(m \geq 1)$, we have $G \in \mathcal{S}_{3}$. This contradiction completes the proof of Theorem 3 .

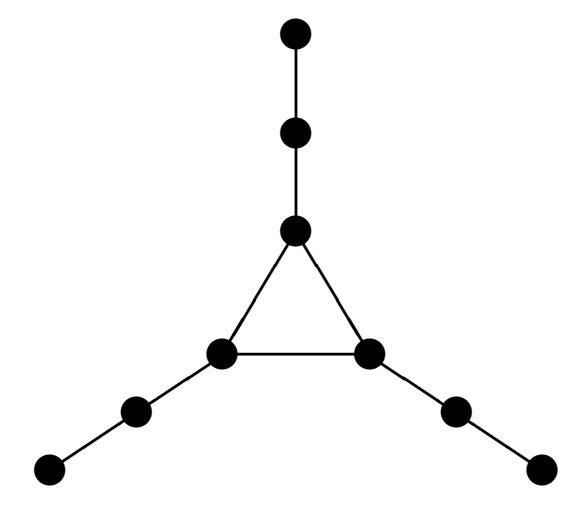

FIGURE 3. Graph $H$ of Corollary 2.

The next result follows directly from Theorem 3 and Theorem A, since each graph from $\mathcal{S}$ contains either $C_{4}$ or the graph $H$ of Fig. 3 as an induced subgraph.

Corollary 2 (Jacobson and Peters [23]) If a graph $G$ does not contain either $K_{1,3}, C_{4}$ or the graph $H$ of Fig. 3 as an induced subgraph, then $G$ is $\Gamma$-perfect and IR-perfect. 
Note in conclusion that using properties of minimal $\Gamma$-imperfect graphs stated in Proposition 2, it is not difficult to prove Theorems B, C, D, E, or F from Section 1.

Acknowledgment The authors thank the referees for valuable suggestions.

\section{References}

[1] R.B. Allan and R. Laskar, On domination and independent domination numbers of a graph. Discrete Math. 23 (1978) 73-76.

[2] N. Biggs, Algebraic Graph Theory, Cambridge University Press (1974).

[3] B. Bollobás and E.J. Cockayne, Graph-theoretic parameters concerning domination, independence, and irredundance. J. Graph Theory 3 (1979) 241-249.

[4] G. Chartrand and L. Lesniak, Graphs 83 Digraphs, Chapman \& Hall, 3rd ed. (1996).

[5] G.A. Cheston and G. Fricke, Classes of graphs for which upper fractional domination equals independence, upper domination, and upper irredundance. Discrete Appl. Math. 55 (1994) 241-258.

[6] G.A. Cheston, E.O. Hare, S.T. Hedetniemi and R.C. Laskar, Simplicial graphs. Congr. Numer. 67 (1988) 105-113.

[7] E.J. Cockayne, O. Favaron, C. Payan and A.G. Thomason, Contributions to the theory of domination, independence and irredundance in graphs. Discrete Math. 33 (1981) 249-258.

[8] E.J. Cockayne, S.T. Hedetniemi and D.J. Miller, Properties of hereditary hypergraphs and middle graphs. Canad. Math. Bull. 21 (1978) 461-468.

[9] E.J. Cockayne and C.M. Mynhardt, The sequence of upper and lower domination, independence and irredundance numbers of a graph. Discrete Math. 122 (1993) 89102.

[10] O. Favaron, Stability, domination and irredundance in a graph. J. Graph Theory 10 (1986) 429-438.

[11] M. Fellows, G. Fricke, S.T. Hedetniemi and D. Jacobs, The private neighbor cube. SIAM J. Discrete Math. 7 (1994) 41-47.

[12] J. Fulman, A note on the characterization of domination perfect graphs. J. Graph Theory 17 (1993) 47-51.

[13] M.C. Golumbic and R.C. Laskar, Irredundancy in circular arc graphs. Discrete Appl. Math. 44 (1993) 79-89.

[14] G. Gutin and V.E. Zverovich, Upper domination and upper irredundance perfect graphs. Discrete Math. 190 (1998) 95-105. 
[15] P.L. Hammer and F. Maffray, Preperfect graphs. Combinatorica 13 (1993) 199-208.

[16] F. Harary, Graph Theory, Addison-Wesley, Reading, MA (1969).

[17] F. Harary and M. Livingston, Characterization of trees with equal domination and independent domination numbers. Congr. Numer. 55 (1986) 121-150.

[18] T.W. Haynes, S.T. Hedetniemi and P.J. Slater, Domination in Graphs: Advanced Topics, Marcel Dekker, Inc., New York (1998).

[19] T.W. Haynes, S.T. Hedetniemi and P.J. Slater, Fundamentals of Domination in Graphs, Marcel Dekker, Inc., New York (1998).

[20] S.T. Hedetniemi, R. Laskar and J. Pfaff, Irredundance in graphs: a survey. Congr. Numer. 48 (1985) 183-193.

[21] M.A. Henning, Irredundance perfect graphs. Discrete Math. 142 (1995) 107-120.

[22] M.S. Jacobson and K. Peters, A note on graphs which have upper irredundance equal to independence. Discrete Appl. Math. 44 (1993) 91-97.

[23] M.S. Jacobson and K. Peters, Chordal graphs and upper irredundance, upper domination and independence. Discrete Math. 86 (1990) 59-69.

[24] R. Laskar and H.B. Walikar, On domination related concepts in graph theory. Lecture Notes in Math. 885 (1981) 308-320.

[25] R. Laskar and J. Pfaff, Domination and irredundance in graphs. Tech. Report 434, Dept. Mathematical Sciences, Clemson Univ., 1983.

[26] R. Laskar and J. Pfaff, Domination and irredundance in split graphs. Tech. Report 430, Dept. Mathematical Sciences, Clemson Univ., 1983.

[27] S. Mitchell and S. Hedetniemi, Edge domination in trees. Congr. Numer. 19 (1977) 489-509.

[28] D.P. Sumner, Critical concepts in domination. Discrete Math. 86 (1990) 33-46.

[29] D.P. Sumner and J.I. Moore, Domination perfect graphs. Notices Am. Math. Soc. 26 (1979) A-569.

[30] J. Topp, Domination, independence and irredundance in graphs. Dissertationes Math. 342 (1995) 99 pp.

[31] J. Topp and L. Volkmann, On graphs with equal domination and independent domination numbers. Discrete Math. 96 (1991) 75-80.

[32] L. Volkmann and V.E. Zverovich, A proof of Favaron's conjecture on irredundance perfect graphs. (submitted)

[33] I.E. Zverovich and V.E. Zverovich, An induced subgraph characterization of domination perfect graphs. J. Graph Theory 20 (1995) 375-395. 